\title{
Enfermedad celiaca materna no diagnosticada y riesgo de tener hijos con bajo peso al nacimiento
}

\author{
G. Solís Sánchez, C. Blanco Cristóbal ${ }^{1}$, A. Suárez González ${ }^{2}$, F. J. Román Llorente², E. Fernández \\ Rodríguez ${ }^{1}$, N. Fernández González, J. L. Solís Sánchez y L. Mier Lobato ${ }^{3}$ \\ Servicios de Pediatría, ${ }^{1}$ Análisis Clínicos, ${ }^{2}$ Digestivo y ${ }^{3}$ Obstetricia. Hospital de Cabueñes (SESPA). Gijón, Asturias
}

\section{RESUMEN}

Objetivo: conocer la prevalencia de enfermedad celiaca en madres de neonatos mayores y menores de 2.500 gramos de peso al nacimiento.

Pacientes: sujetos: casos: madres de neonatos menores de $2.500 \mathrm{~g}$ al nacimiento. Controles: madres de neonatos mayores de $2.500 \mathrm{~g}$ al nacimiento. Un control por cada caso. Instrumentalización: encuesta clínico-epidemiológica y serología celiaca.

Métodos: estudio caso-control. Ámbito: mujeres del Área Sanitaria V (Gijón) del Principado de Asturias que den a luz en el Hospital de Cabueñes.

Resultados: se analizaron 1.103 mujeres: 577 madres de niños menores de $2.500 \mathrm{~g}$ y 526 madres de niños mayores de $2.500 \mathrm{~g}$. Se diagnosticaron 4 casos de celiaquía, 2 en cada grupo. De las 4 madres, 3 dieron a luz a niños a término (1 caso cada 235 madres, prevalencia $0,42 \%$ ) y 1 dio a luz a un niño prematuro ( 1 caso cada 389 madres, prevalencia 0,26\%). Dos madres dieron a luz a niños de peso adecuado para su edad gestacional (1/419 madres, prevalencia $0,24 \%$ ) y 2 madres dieron a luz niños de bajo peso para su edad gestacional (1/132 madres, prevalencia $0,75 \%)$. La odds ratio para peso menor de $2.500 \mathrm{~g}$ al nacimiento fue 0,91 (IC 95\% 0,126,49 ), para prematuridad 0,61 (IC 95\% 0,06-5,89) y para bajo peso para su edad gestacional $3,19(0,44-22,79)$.

Conclusiones: la prevalencia de enfermedad celiaca en mujeres fértiles de Gijón es de un caso cada 275 madres (prevalencia $0,36 \%)$, sin que hayamos encontrado diferencias entre los dos grupos estudiados.

Palabras clave: Enfermedad celiaca. Recién nacido. Bajo peso al nacimiento.

\begin{abstract}
Aims: in order to know the prevalence of celiac disease in mothers with newborns weighing less or more than 2,500 g at birth we carried out a case-control study.

Patients: mothers of newborns in Cabueñes Hospital. Case group: Mothers with babies weighing less than 2,500 g at birth. Controls: Mothers with babies weighing more than 2,500 g at birth. One control for each case.

Methods: epidemiological and clinical interviews, and celiac disease serology.

Results: we studied 1103 women: 577 cases and 526 controls. We diagnosed 4 celiac disease cases, 2 in the case group and 2 in the control group. These 4 mothers had 3 term newborns ( 1 case in each 235 mothers; prevalence $0.42 \%$ ) and 1 preterm newborn ( 1 case in each 389 mothers; prevalence $0.26 \%)$. Two cases had babies with adequate birth weight for their gestational age ( 1 case in each 419 mothers; prevalence $0.24 \%$ ) and two cases had babies with low birth weight for their gestational age ( 1 in each 132 mothers; prevalence $0.75 \%$ ). The odds ratio for low birth weight was 0.91 (95\% CI: 0.12-6.49), the odds ratio for preterm birth was 0.61 (95\% CI: 0.06-5.89), ad the odds ratio for low birth weight for gestational age was 3.19 (95\% CI: 0.4422.79).

Conclusions: the prevalence of celiac disease in fertile women in our geographic area was $0.36 \%$ (1 case in each 275 mothers), and no differences were found between study groups.
\end{abstract}

Key words: Celiac disease. Newborn. Low birth weight.

Solís Sánchez G, Blanco Cristóbal C, Suárez González A, Román Llorente FJ, Fernández Rodríguez E, Fernández González N, Solís Sánchez JL, Mier Lobato L. Enfermedad celiaca materna no diagnosticada y riesgo de tener hijos con bajo peso al nacimiento. Rev Esp Enferm Dig 2008; 100: 332-336.

*Este estudio ha sido financiado con la beca del Fondo de Investigaciones Sanitarias FIS PI021422.

Recibido: 23-01-08.

Aceptado: 26-02-08.

Correspondencia: G. Solís. Servicio de Pediatría. Hospital de Cabueñes. Cabueñes, s/n. 33394 Gijón, Asturias.e-mail: gsolis@telefonica.net

\section{INTRODUCCIÓN}

La prevalencia de enfermedad celiaca en la población general varía de forma importante de unos trabajos a otros. Actualmente, se acepta que podría afectar al 0,5$1 \%$, mientras que sólo uno de cada ocho casos se diagnostica. En el adulto, como en el niño, la presentación clí- 
nica digestiva clásica cada vez es más infrecuente y la mayoría de los nuevos casos de enfermedad celiaca se diagnostican por cribados poblacionales, por formas oligosintomáticas o en el curso de un estudio por otro tipo de patología (1-6).

Son muchos y muy variados los problemas de salud que se asocian a la celiaquía por compartir con ella mecanismos inmunopatogénicos y/o por ser secundarios a la malabsorción intestinal que produce. En la mujer, la enfermedad celiaca ha sido asociada a alteraciones obstétricas y ginecológicas, como infertilidad, abortos de repetición, bajo peso al nacimiento, menor duración de la lactancia materna, entre otros. Sin embargo, los datos de las series publicadas no siempre coinciden en estas apreciaciones (6-15).

Con las cifras de prevalencia de enfermedad celiaca que se publican y si esta fuese causa de bajo peso al nacimiento en las mujeres afectas no tratadas, podría ser coste-efectivo y muy útil clínicamente el cribado de esta enfermedad en las mujeres antes de la gestación, con vistas a recomendar su tratamiento a las enfermas y evitar este factor como causa de bajo peso al nacimiento.

Nosotros planteamos un estudio caso control sobre la relación entre el bajo peso al nacimiento y la enfermedad celiaca materna no diagnosticada, con el fin de valorar la posible asociación entre ambos.

\section{MATERIAL Y MÉTODOS}

Estudio epidemiológico, no experimental, analítico, caso-control, prospectivo. El diseño consta de dos partes: la primera epidemiológica, en forma de una entrevista personal a madres, y la segunda de laboratorio, mediante la determinación en una muestra de sangre materna postparto de marcadores de enfermedad celiaca (anticuerpos antiendomisio y antitransglutaminasa).

El estudio se realizó en el Área Sanitaria V del Principado de Asturias (concejos de Carreño, Gijón y Villaviciosa) en España. Dicha área sanitaria tiene una población predominantemente urbana $(90 \%$ en la ciudad de Gijón), de 300.000 habitantes, de los cuales 150.000 son mujeres, estando el 25\% en edad fértil (15-49 años).

Denominamos casos a las madres de los recién nacidos vivos que durante este periodo de tiempo pesaran menos de $2.500 \mathrm{~g}$ de peso al nacimiento. Los controles fueron madres de los recién nacidos vivos de peso mayor de $2.500 \mathrm{~g}$. Casos y controles se tomaron en paralelo (lo más cercanos posible), aunque no apareados, un control por cada caso. El periodo de recogida de datos fue de 3 años (2003-2005). Se solicitó permiso explícito a todas las madres para incluirlas en el estudio. A todas se les realizó la entrevista y, simultáneamente a la extracción del hemograma postparto, se tomó una muestra de sangre para determinación analítica en suero. El estudio fue financiado por el Fondo de Investigaciones Sanitarias (FIS PI021422) del Estado español y contó con la aprobación del Comité de Ética en Investigación del Principado de Asturias.

En la entrevista epidemiológica se solicitaba información, mediante encuesta de preguntas cerradas, sobre filiación, datos epidemiológicos básicos, antecedentes patológicos maternos, historia obstétrica materna, historia clínica de gestación actual y datos sobre el recién nacido. Los entrevistadores (tres médicos) fueron entrenados para seguir criterios homogéneos. La entrevista se realizó 24-48 horas tras el parto, de forma personal y privada. Se aseguró la absoluta confidencialidad de los datos obtenidos.

En los sueros extraídos a cada paciente se determinó: anticuerpos antitransglutaminasa tisular clase A (antitTG), inmunoglobulinas tipo A totales ( $\operatorname{IgA}$ ) y anticuerpos antiendomisio (AEA o EMA) de clase IgA. En caso de existir alguna muestra con deficiencia de IgA, se determinaron los anticuerpos antiendomisio del tipo IgG. Para la determinación de los anti-tTG por la técnica de ELISA, se utilizó el kit comercial Celikey TM Tissue Transglutaminase (human, recombinant) IgA Antibody Assay (PHARMACIA Diagnostics ${ }^{\circledR}$ ). Para la determinación cuantitativa de las inmunoglobulinas IgA, se utilizó el sistema BN ( $\mathrm{N}$ antisueros contra las inmunoglobulinas humanas) de Dade Behring Marburg GMBH ${ }^{\circledR}$. Para determinar los anticuerpos antiendomisio de clase A y $G$ por inmunofluorescencia indirecta se emplearon secciones de esófago de mono (anticuerpos antiendomisio -AEA-, Biosystems $\left.{ }^{\circledR}\right)$.

Las mujeres con serología positiva para ambos marcadores (anticuerpos antiendomisio y antitransglutaminasa tisular) fueron estudiadas en la Consulta Externa de Digestivo, con objeto de confirmar el diagnóstico. Se les propuso la realización de biopsia duodenal, mediante la técnica de gastroduodenoscopia, con toma de muestras múltiples.

Todas las entrevistas fueron introducidas en una base de datos, para posteriormente, y tras su validación, ser analizados en un programa informático estadístico (SPSS $\left.\mathrm{PC}+{ }^{\circledR} 10.0\right)$, con licencia de uso. En el estudio matemático inicialmente se realizó un análisis descriptivo de todas las variables y, posteriormente, una comparación univariante entre casos y controles.

\section{RESULTADOS}

\section{Análisis de la serie}

Se estudiaron 1.103 madres: 577 madres de niños menores de $2.500 \mathrm{~g}$ al nacimiento y 526 madres de niños mayores de $2.500 \mathrm{~g}$ al nacimiento. De estas 1.103 madres nacieron 1.200 niños (1.009 partos sencillos, 88 gemelares y 5 triples): 465 prematuros (menores de 37 semanas de edad gestacional), 733 a término (37 a 42 semanas de edad gestacional) y 2 postérmino (más de 42 semanas de edad gestacional). La distribución del peso de los niños al 
nacimiento fue la siguiente: 625 menores de 2.500 gramos y 575 mayores de 2.500 gramos. De los 1.200 niños, 264 eran bajo peso para su edad gestacional (menores del percentil 10 para su edad gestacional).

Las madres tenían una edad media de 31,1 años (IC 95\% 30,9-31,5 años) y el 92,7\% eran nacidas en España. El $41 \%$ tenía estudios básicos, el $24,8 \%$ había terminado sus estudios de bachillerato y el $34,1 \%$ tenía estudios universitarios. No hubo diferencias por edad ni por origen entre los casos y los controles, pero sí en cuanto al nivel de estudios: los estudios elementales se asociaron significativamente con el bajo peso (OR 1,54 [IC 95\% 1,21$1,96])$.

Un 30,8\% tenía antecedentes personales patológicos, destacando problemas dermatológicos en el $15,2 \%$, respiratorios en el $8,3 \%$, endocrinológicos en el 3,7\%, neurológicos en el 2,9\%, hematológicos en el 2,9\%, cardiovasculares en el $1,9 \%$, infecciosos en el $2,1 \%$, digestivos en el 2,5\%, ginecológicos en el 2,3\%, renales en el 1,5\% y psiquiátricos en el 1,2\%. El 2,1\% eran toxicómanas. Seis madres habían sido prematuras. El 2\% (22 madres) tenía antecedentes familiares de enfermedad celiaca en familiares de primer y segundo grado. En la tabla I se pueden leer los odds ratio (OR) calculados para los distintos tipos de patología materna en relación al bajo peso al nacimiento.

\begin{tabular}{|c|c|c|c|}
\hline & Casos & $\begin{array}{l}\text { Grupo } \\
\text { control }\end{array}$ & $\begin{array}{c}\text { OR } \\
(\text { IC } 95 \%)\end{array}$ \\
\hline Antecedentes personales de prematuridad & 4/577 & 2/525 & $1,8(0,3-10,0)$ \\
\hline $\begin{array}{l}\text { Antecedentes patológicos maternos } \\
\text { Enf. infecciosas } \\
\text { Enf. respiratorias } \\
\text { Enf. hematológicas } \\
\text { Enf. ap. locomotor } \\
\text { Enf. cardiovasculares } \\
\text { Enf. endocrinas } \\
\text { Enf. renales } \\
\text { Enf. digestivas } \\
\text { Enf. psiquíátricas } \\
\text { Enf. neurológicas } \\
\text { Enf. ginecológicas } \\
\text { Enf. ORL } \\
\text { Enf. reumáticas } \\
\text { Toxicomanía }\end{array}$ & $\begin{array}{l}196 / 577 \\
18 / 577 \\
44 / 577 \\
14 / 577 \\
6 / 577 \\
16 / 577 \\
22 / 577 \\
11 / 577 \\
16 / 577 \\
13 / 577 \\
20 / 577 \\
15 / 577 \\
1 / 577 \\
4 / 577 \\
19 / 577\end{array}$ & $\begin{array}{l}144 / 525 \\
5 / 525 \\
47 / 525 \\
18 / 525 \\
5 / 525 \\
5 / 525 \\
19 / 525 \\
6 / 525 \\
12 / 525 \\
1 / 525 \\
12 / 525 \\
10 / 525 \\
5 / 525 \\
4 / 525 \\
4 / 525\end{array}$ & $\begin{array}{l}1,3(1,05-1,7) \\
3,3(1,2-9,0) \\
0,8(0,5-1,3) \\
0,7(0,3-1,4) \\
1,1(0,3-3,6) \\
2,9(1,0-8,1) \\
1,0(0,5-1,9) \\
1,6(0,6-4,5) \\
1,2(0,5-2,5) \\
1,7(1,0-290) \\
1,5(0,7-3,1) \\
1,3(0,6-3,0) \\
0,1(0,02-1,5) \\
0,9(0,2-3,6) \\
6,9(1,5-43,5)\end{array}$ \\
\hline
\end{tabular}

La edad media de la menarquia fue de 12,6 años (IC 95\% 12,5-12,7 años) y el 21,8\% relataba alteraciones en su ciclo menstrual. El $49 \%$ de la serie tenía gestaciones previas, el 28,3\% abortos previos (el 10,5\% interrupciones voluntarias del embarazo), el 43,9\% hijos previos y el $9,3 \%$ hijos de menos de $2.500 \mathrm{~g}$ al nacimiento previos. El $5,7 \%$ tenía problemas de fertilidad y había recibido tratamiento por este motivo.
Las madres de los niños de peso menor de $2.500 \mathrm{~g}$ presentaban mayor frecuencia de alteraciones en la menstruación (OR 1,3 [IC 95\% 1,04-1,8]) y de historia de tratamiento para fertilidad (OR 4,6 [IC 95\% 2,3-9,0]) que las de los niños de peso mayor de 2.500 gramos al nacimiento.

Durante esta gestación, el 68\% había trabajado fuera de casa y el 33,3\% fumaba tabaco. El consumo de tabaco aumentó significativamente el riesgo de tener hijos con bajo peso (OR 1,9 [IC 95\% 1,4-2,4]).

De las gestaciones analizadas, el 16,7\% presentó rotura prematura de membranas, el $14,1 \%$ crecimiento intrauterino retardado, el 12,4\% hipertensión arterial, el 8,4\% gestación múltiple, el 8,3\% infección urinaria en el tercer trimestre, el 7,8\% amenaza de parto prematuro, el 7,6\% amenaza de aborto y el $4,7 \%$ diabetes gestacional.

\section{Casos diagnosticados de celiaquía}

De las 1.103 madres, 4 presentaron anticuerpos antitransglutaminasa y antiendomisio positivos, siendo diagnosticadas de enfermedad celiaca $(0,36 \%, 1$ cada 275 madres). Tres fueron confirmadas por biopsia, mientras que la cuarta había sido diagnosticada por biopsia en su infancia, pero había abandonado el tratamiento dietético durante su juventud y ya se creía curada, negándose a repetir su biopsia y aceptando el diagnóstico.

Los cuatro casos de enfermedad celiaca diagnosticados correspondieron dos al grupo de madres de niños menores de $2.500 \mathrm{~g}(0,34 \%)$ y dos al grupo de madres de niños mayores de $2.500 \mathrm{~g}(0,38 \%)$.

De las 4 madres, 3 dieron a luz a niños a término ( 1 caso cada 235 madres, prevalencia $0,42 \%$ ) y una a un niño prematuro ( 1 caso cada 389 madres, prevalencia $0,26 \%$ ). Dos niños fueron de peso adecuado a su edad gestacional (1 caso cada 419 madres, prevalencia $0,24 \%$ ) y dos pequeños para la edad estacional (uno nacido a término y otro prematuro) (1 caso cada 132 madres, prevalencia $0,75 \%$ ).

La odds ratio para peso menor de $2.500 \mathrm{~g}$ al nacimiento fue 0,91 (IC 95\% 0,12-6,49), para prematuridad 0,61 (IC $95 \% 0,06-5,89)$ y para bajo peso para su edad gestacional 3,19 (IC 95\% 0,44-22,79).

Siete madres presentaron en su analítica déficit de inmunoglobulina $\mathrm{A}(0,6 \%, 1$ cada 157 madres), pero ninguna de ellas presentó anticuerpos antiendomisio IgG positivos.

En la tabla II se pueden leer las características principales de las cuatro madres diagnosticadas. Dos madres presentaban anemia y/o ferropenia, mientras que las otras dos estaban asintomáticas (una de ellas era la celiaca diagnosticada en la infancia que había abandonado el tratamiento).

\section{DISCUSIÓN}

La enfermedad celiaca no diagnosticada ni tratada puede afectar a mujeres en sus años fértiles y, por lo tanto, la malabsorción que produce puede interferir con la 
Tabla II. Características de las cuatro madres diagnosticadas de enfermedad celiaca en nuestra serie

\begin{tabular}{lcccc}
\hline & $\begin{array}{c}\text { Pneonato/ } \\
\text { Edad gestacional }\end{array}$ & $\begin{array}{c}\text { Gestaciones } \\
\text { previas }\end{array}$ & $\begin{array}{c}\text { Abortos } \\
\text { previos }\end{array}$ & Comentario \\
\hline Madre $n^{\circ} 1$ & $2.420 / 38$ & 0 & 0 & Asintomática \\
Madre $n^{\circ} 2$ & $3.490 / 39$ & 2 & 1 & Anemia crónica \\
Madre $n^{\circ} 3$ & $2.600 / 35$ & 1 & 1 & Ferropenia crónica \\
Madre $n^{\circ} 4$ & $2.410 / 40$ & 3 & 1 & Celiaca en la infancia \\
\hline
\end{tabular}

embriogénesis, nutrición y crecimiento fetal en caso de embarazo $(12,13)$.

Nuestro estudio pretendía conocer si existía relación entre la enfermedad celiaca no diagnosticada ni tratada y el nacimiento de niños con peso menor de $2.500 \mathrm{~g}$, ya que, si esta relación existiera, podría ser interesante realizar un cribado de celiaquía a todas las mujeres antes o durante el primer trimestre de la gestación. Para conseguir nuestro objetivo, realizamos un estudio de casos y controles en un hospital público del norte de España. Aunque el diseño de casos y controles no es el tipo ideal para valorar causalidad, entre todos los esquemas posibles de estudio elegimos este por tener un buen rendimiento económico y temporal dada la incidencia del problema en nuestro medio.

El resultado principal de nuestro trabajo indica que la pre- valencia de enfermedad celiaca en mujeres fértiles de nuestra zona es de una enferma cada 275 mujeres, sin que hayamos encontrado diferencias entre las madres de niños de más o menos de $2.500 \mathrm{~g}$ de peso al nacimiento. Por subgrupos de análisis, la única asociación clínicamente valorable, aunque estadísticamente no significativa, es la de celiaquía materna y neonato de bajo peso para su edad gestacional.

El bajo peso al nacimiento es un importante problema médico y social, con una variada lista etiológica. La relación entre el bajo peso al nacimiento y la enfermedad celiaca materna no diagnosticada ha sido analizada por distintos investigadores en los últimos años (Tabla III).

Se ha publicado que la enfermedad celiaca no diagnosticada se asocia en la mujer a múltiples problemas obstétricos y ginecológicos. Algunos trabajos apuntan a que las mujeres con enfermedad celiaca tienen un acortamiento de su vida fértil con un retraso en la menarquia y una menopausia adelantada respecto a mujeres sanas. También que los problemas de fertilidad son comunes tanto en hombres como mujeres con enfermedad celiaca y pueden ser el primer síntoma de la enfermedad. Otros estudios han señalado que existe una mayor frecuencia de mortinatos y abortos entre mujeres celiacas no diagnosticadas. Por último, se ha publicado que estas mujeres no diagnosticadas ni tratadas tienen un mayor riesgo de tener hijos de bajo peso que la población general (8-17). Sin embargo estos hallazgos han sido puestos en duda en los últimos años por algunos autores (15).

Tabla III. Trabajos epidemiológicos publicados más importantes sobre la relación entre enfermedad celiaca materna y riesgos para los hijos

\begin{tabular}{|c|c|c|c|c|}
\hline Autor & Revista y año & Población & País & Conclusión \\
\hline Ciaccic & Am J Gastroenterol 1996 & $\begin{array}{l}94 \text { madres celiacas no tratadas y } \\
34 \text { madres celiacas tratadas }\end{array}$ & Italia & $\begin{array}{l}\text { Celiacas no tratadas: mayor riesgo de aborto, bajo peso } \\
\text { y lactancia materna más corta }\end{array}$ \\
\hline Sher KS & Acta Paediatr 1996 & $\begin{array}{l}80 \text { madres celiacas y } 70 \text { madres } \\
\text { no celiacas }\end{array}$ & Reino Unido & $\begin{array}{l}\text { Celiacas: menarquia tardía, más mortinatos y muertes } \\
\text { perinatales }\end{array}$ \\
\hline Norgard B & Am J Gastroenterol 1999 & 127 mujeres celiacas & Dinamarca & $\begin{array}{l}\text { Celiacas no tratadas: mayor riesgo de bajo peso y de crecimiento in- } \\
\text { trauterino retardado } \\
\text { Celiacas tratadas: sin diferencias con población general }\end{array}$ \\
\hline Martinelli P & Gut 2000 & 845 mujeres, 12 de ellas celiacas & Italia & Celiaca: resultados desfavorables de la gestación \\
\hline Gasbarrini A & Lancet 2000 & $\begin{array}{l}44 \text { mujeres con abortos repetición, } \\
39 \text { mujeres con crecimiento } \\
\text { intrauterino retardado y } \\
50 \text { donantes sanas }\end{array}$ & Italia & $\begin{array}{l}\text { Mujeres con abortos de repetición o con crecimiento } \\
\text { intrauterino retardado: mayor riesgo de ser celiacas }\end{array}$ \\
\hline Ludvigsson JF & Gut 2001 & $\begin{array}{l}10.500 \text { madres y padres ( } 53 \text { madres } \\
\text { y } 27 \text { padres celiacos) }\end{array}$ & Suecia & $\begin{array}{l}\text { Hijos de madres y padres celiacos, tratados o no: mayor riesgo } \\
\text { de prematuridad y bajo peso }\end{array}$ \\
\hline Greco L & Gut 2004 & 5.000 mujeres & Italia & Celiaca: no asociación a resultados desfavorables de la gestación \\
\hline Tata L & Gastroenterology 2005 & $\begin{array}{l}1.500 \text { mujeres celiacas y } \\
7.700 \text { mujeres no celiacas }\end{array}$ & Reino Unido & $\begin{array}{l}\text { Fertilidad igual en ambos grupos. Riesgo de cesárea y aborto } \\
\text { ligeramente superior en celiacas }\end{array}$ \\
\hline Lludvigsson JF & Gastroenterology 2005 & 2.078 mujeres celiacas & Suecia & $\begin{array}{l}\text { Mayor riesgo de crecimiento intrauterino retardado, bajo peso, } \\
\text { prematuridad y cesárea. Riesgo desaparece con tratamiento }\end{array}$ \\
\hline Salvatores & Am J Gastroenterol 2007 & $\begin{array}{l}1.714 \text { padres y madres de niños } \\
\text { prematuros y de bajo peso para su } \\
\text { edad gestacional }\end{array}$ & Italia & $\begin{array}{l}\text { La prevalencia de celiaquía en madres de niños de bajo peso para } \\
\text { su edad gestacional es mayor que en población normal }\end{array}$ \\
\hline
\end{tabular}


Nuestros datos, con las limitaciones del diseño de casos y controles, muestran dos conclusiones. En primer lugar, la frecuencia de enfermedad celiaca no diagnosticada en nuestro medio entre mujeres fértiles es de un caso cada $275 \mathrm{mu}-$ jeres, cifra bastante inferior a la propuesta por otros autores, pero cercana a la publicada previamente en nuestro medio (18). Y en segundo lugar, no encontramos asociación entre la enfermedad celiaca materna y el peso al nacimiento menor de $2.500 \mathrm{~g}$ o la prematuridad. Nuestras cuatro madres celiacas no tratadas se repartieron por igual entre ambos grupos de estudio: dos madres de niños de peso menor de 2.500 gramos y dos de niños mayores de ese peso. Tan sólo encontramos una asociación valorable entre los niños de bajo peso para su edad gestacional y la celiaquía materna, si bien no se alcanzó la significación estadística. Pero, además, en contra de lo publicado previamente, ninguna de nuestras cuatro madres celiacas presentaba problemas de esterilidad, ni abortos de repetición ni alteraciones en la menstruación.

\section{BIBLIOGRAFÍA}

1. Van Heel DA, West J. Recent advances in coeliac disease. Gut 2006; 55: $1037-46$.

2. Greco L, Veneziano A, Di Donato L, Zampella C, Pecoraro M, Paladini $\mathrm{D}$, et al. Undiagnosed celiac disease does not appear to be associated with unfavourable outcome of pregnancy. Gut 2004; 53: 14951.

3. Casellas i Jordá F. Enfermedad celiaca. Med Clin (Barc) 2006; 126: 137-42.

4. García Novo MD, García C, Acuña Quirós MD, Asensio J, Zancada G, Barrio Gutiérrez S, et al. Prevalencia de enfermedad celiaca en donantes de sangre aparentemente sanos en la comunidad autónoma de Madrid. Rev Esp Enferm Dig 2007; 99: 337-42.
5. Rodrigo L, Fuentes D, Riestra S, Niño P, Álvarez N, López-Vázquez $\mathrm{A}$, et al. Increased prevalence of celiac disease in first and secondgrade relatives. A report of a family with 19 studied members. Rev Esp Enferm Dig 2007; 99: 149-55.

6. Cantarero Vallejo MD, Gómez Camarero J, Manchén L, Pajares Díaz JA, Lo Iacono O. Liver damage and celiac disease. Rev Esp Enferm Dig 2007; 99: 648-52.

7. Farrell RJ, Kelly CP. Celiac sprue. N Engl J Med 2002; 346: 180-8.

8. Green PHR, Jabri B. Coeliac disease. Lancet 2003; 362: 383-91.

9. Sher KS, Mayberry JF. Female fertility, obstetric and gynaecological history in celiac disease: A case control study. Acta Paediatr 1996; (Supl. 412): 76-7.

10. Norgard B, Fonager K, Sorensen HT, Olsen J. Birth outcomes of women with celiac disease: A national historical cohort study. Am J Gastroenterol 1999; 94: 2435-40.

11. Ciacci C, Cirillo M, Auriemma G, Di Dato G, Sabbatini F, Mazzacca G. Celiac disease and pregnancy outcome. Am J Gastroenterol 1996; 91: 718-22.

12. Martinelli P, Troncone R, Paparo F, Torre P, Trapanese E, Fasano C, et al. Coeliac disease and unfavourable outcome of pregnancy. Gut 2000; 46: 332-5.

13. Ludvigsson JF, Montgomery SM, Ekbom A. Celiac disease and risk of adverse fetal outcome: A population-based cohort study. Gastroenterology 2005; 129: 454-63.

14. Tata LJ, Card TR, Logan RFA, Hubbard RB, Smith CJP, West J. Fertility and pregnancy-related events in women with celiac disease: A population-based cohort study. Gastroenterology 2005; 128: 84955 .

15. Salvatore S, Finazzi S, Radaelli G, Lotzniker M, Zuccotti GV, on behalf of Premacel Study Group. Prevalence of undiagnosed celiac disease in the parents of preterm and/or small for gestational age infants. Am J Gastroenterol 2006; 102: 168-73.

16. Gasbarrini A, Sanz Torre E, Trivellini C, De Carolis S, Caruso A, Gasbarrini G. Recurrent spontaneous abortion and intrauterine fetal growth retardation as symptoms of celiac disease. Lancet 2000; 356: 399-400.

17. Ludvigsson JF, Ludvigsson J. Coeliac disease in the father affects the newborn. Gut 2001; 49: 169-75.

18. Riestra S, Fernández E, Rodrigo L, García S, Ocio G. Prevalence of coeliac disease in the general population of northern Spain. Strategies of serologic screening. Scand J Gastroenterol 2000; 35: 398-402. 